\title{
The pursuit of a dream, Francisco Javier Sayas and the HDG methods
}

\author{
Bernardo Cockburn ${ }^{1}$ (D)
}

Received: 12 July 2021 / Accepted: 2 October 2021 / Published online: 16 October 2021

(c) The Author(s) 2021

\begin{abstract}
Franciso Javier Sayas, man of grit and determination, left his hometown of Zaragoza in 2007 in pursuit of a dream, to become a scholar in the USA. I hosted him in Minneapolis, where he spent three long years of an arduous transition before obtaining a permanent position at the University of Delaware. There, he enthusiastically worked on the unfolding of his dream until his life was tragically cut short by cancer, at only 50. In this paper, I try to bring to light the part of his academic life he shared with me. As we both worked on hybridizable discontinuous Galerkin methods, and he wrote a book on the subject, I will tell Javier's life as it developed around this topic. First, I will show how the ideas of static condensation and hybridization, proposed back in the mid $60 \mathrm{~s}$, lead to the introduction of those methods. This background material will allow me to tell the story of the evolution of the hybridizable discontinuous Galerkin methods and describe Javier's participation in it. Javier faced death with open eyes and poised dignity. I will end with a poem he liked.
\end{abstract}

Keywords Discontinuous Galerkin · Static condensation · Hybridization · Postprocessing · Superconvergence $\cdot$ M-decompositions

Mathematics Subject Classification 65N30

\section{Prologue: numerical analysis \& no regrets}

Sensing that his death was close, Francisco Javier Sayas organized, on Saturday February 16th, 2019, a farewell, 1-day meeting he entitled Numerical Analysis \& No Regrets. Since Javier did like the talk I gave on that occasion, I gave a slightly expanded version of it at the tribute in his honor organized by the Spanish Society for Applied Mathematics at the XXVI Congreso de Ecuaciones Diferenciales y Applicaciones/XVI Congreso de Matemática Aplicada, June 14-18, 2021, Gijón, Spain. This paper captures that talk.

Supported in part by the National Science Foundation through DMS Grant 1912646.

$凶$ Bernardo Cockburn

cockburn@math.umn.edu

1 School of Mathematics, University of Minnesota, Minneapolis, MN 55455, USA 


\section{Javier's three triptychs}

We all know of Hieronymus Bosch's famous triptych, The Garden of Earthly Delights, 1490-1510, whose original hangs at the Museo del Prado, Madrid. Its many scenes seem to be expecting us to click on them to unzip the stories they are ready to reveal. I did not find any better way to illustrate Javier's academic life than by using triptychs.

\subsection{Javier's academic life triptych}

The first triptych is unconventionally vertical, see Fig. 1, and is about the three main periods of Javier's life: The Zaragoza, the Minneapolis and the Delaware periods.

Javier's Zaragoza period starts in 1994, when he got his Ph.D. in Mathematics under the direction of the prestigious mathematician Michel Crouzeix from the University of Rennes. The period ends in November 2007, when he left his hometown of Zaragoza. I did not know much about Javier during that time except that, in January 10th of 2006, I met him in Concepción, Chile, as we can see in the top panel: From right to left, Rommel Bustinza, Javier Sayas, the author, Gabriel Gatica (collaborator and friend of Javier's), Maggie (Gabriel's wife) and a lady whose name I cannot remember anymore. We were enjoying Chilean hospitality in the restaurant Sólo Carnes after a hard working day in the 2006 Third Summer School organized by the Department of Mathematical Engineering of the Universidad de Concepción. On that occasion, Javier gave a course on Boundary Element methods, certainly his favorite subject in Numerical Analysis.

Javier's Minneapolis period runs from November 2007 until August 2010, time during which he was a Visiting Associate Professor at the School of Mathematics of the University of Minnesota. In the middle panel, we can see Javier enjoying a well deserved beer. (Yes, the temperature is not always -30 degrees centigrade in Minnesota!) He was in the backyard of my house, where we were having what we call the Numerical Analysis Seminar BBQ. Not in the picture were the other participants of the seminar, Ph.D. students, postdocs and visitors, who were taking proper care of the bratwursts with sauerkraut and other delights.

Javier's Delaware period began in August 2010, when he went to the University of Delaware with a tenure-track position of Associate Professor-he became Full Professor with tenure in 2013. It ended tragically on April 2, 2019, when he died from cancer. The last panel is a picture of a dinner during the IMA workshop entitled Recent Advances and Challenges in Discontinuous Galerkin Methods and Related Approaches, from June 29 to July 1, 2017, where Javier gave an invited talk entitled Waves in viscoelastic media. In the picture are, from the right and around the table, Jay Gopalakrishnan, the author, Nilima Nigam, Rosario Grau, Javier Sayas, and a participant of the IMA workshop my facial recognition algorithm fails to identify. We can see Javier in his best shape: poised and confident. Clearly, he had been invigorated by his work on the many aspects that academic life offers in the USA: doing research, organizing national and international meetings and minisymposia, training Ph.D. students, being the Director of Graduate Studies, and, sure enough, practicing the lovely art of writing grant proposals. If we click on Javier's picture, we can unzip his feeling of a sense of belonging to a scientific community that embraced and respected him as a mathematician. 

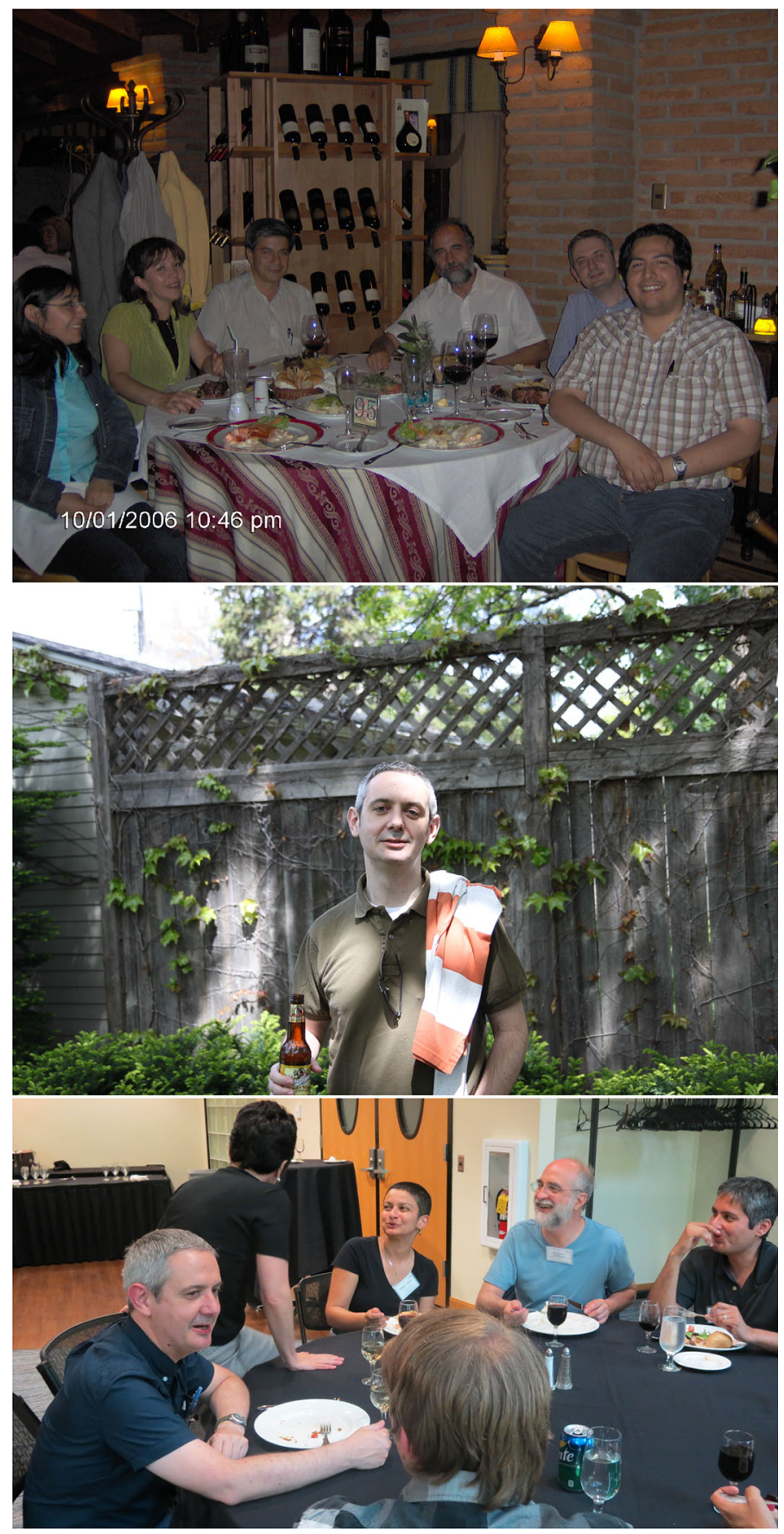

Fig. 1 Javier's academic life triptych: The Zaragoza period 1994-2007 (top), the Minneapolis period 20072010 (middle) and the Delaware period 2010-2019 (bottom) 


\subsection{Javier's Minneapolis triptych}

The second triptych is about Javier's life in Minneapolis.

The top panel depicts El descubrimiento de América por Cristóbal Colón, oil on canvas by Salvador Dalí, 1958/59. The many crosses are Javier's Western, Christian cultural forces which propelled him across the Atlantic. And Gala stands for the promise of tenure in the American New World. Javier arrives to the New Continent with the anticipation of the encounter with an academic El Dorado and a trepidation, for stepping into uncharted territory, somewhat assuaged by a little help from his friends: Gabriel Gatica, from the University of Concepción, and Tomás Chacón, from the University of Sevilla, had alerted me about Javier's transatlantic venture. Harry Singh, from the School of Mathematics of the University of Minnesota, Rosario Grau (my wife) and I were ready to host him in Minneapolis (Fig. 2).

The panel of the middle is Tránsito en espiral, oil on canvas by Remedios Varo, 1962. Remarkable woman of strong political convictions, Remedios Varo was a student of G.I. Gurdieff's ideas and one of the main surrealist painters of her time. Like Javier, she was Spaniard, immigrated to the New World (México), enjoyed 9 years of success as an artist and also died tragically, at 54.

Remedios Varo could very well understand all the works and tribulations that assail an immigrant. Few have seen the actual Wall, the energy barrier to the completion of a transitional but long, sustained and difficult task, as well as Remedios Varo did. As we see in this panel, from his basis in Minneapolis, Javier put three long years to circumnavigate the Wall with great effort, grit and determination. Note how Javier is pedaling furiously strange-looking vehicles along the channel made by the spiraling wall. Some of them are papers by himself, like the 2009 SINUM paper [59] whose main result was celebrated by re-publishing the paper later in 2016 in the prestigious SIAM Review [60]. Others are papers with his new colaborators Jay Gopalakrishnan, Johnny Guzmán, Youngmock Jeon, Ngoc-Cuong Nguyen, Jaime Peraire and Manuel Solano. Yet others, those smaller and closer to the tower of the center, are his no less relevant job interview talks.

The panel of the bottom shows Saint George defeating the Dragon, by Johann König, 1630. It signals that the spiraling iterations around the tower are over and that the desired goal is finally achieved. We see Javier giving a great interview talk at the University of Delaware thus defeating the dark forces that had been barring his entrance to the world of scholars in the USA. On the left and behind him, a crowned tenure-track is waiting for him. At this point, Javier knew that his dream would be realized (Fig. 3).

\subsection{Javier's Delaware triptych}

The third and last triptych has the classical configuration and displays the covers of the three books that Javier wrote and published during his tenure at the University of Delaware. They are a reflection of Javier's passion for the craft of clarification and mathematical exposition, and represent the rich academic life that Javier was able to realize.

The book on the right, written with his then Ph.D. student Shukai Du, is about the socalled hybridizable discontinuous Galerkin (HDG) methods [38]. It was completed only a few weeks before Javier's death. To honor Javier's interest in the HDG methods, a favorite topic of mine, in what follows, I describe these methods and tell the story of how Javier participated in their evolution. 


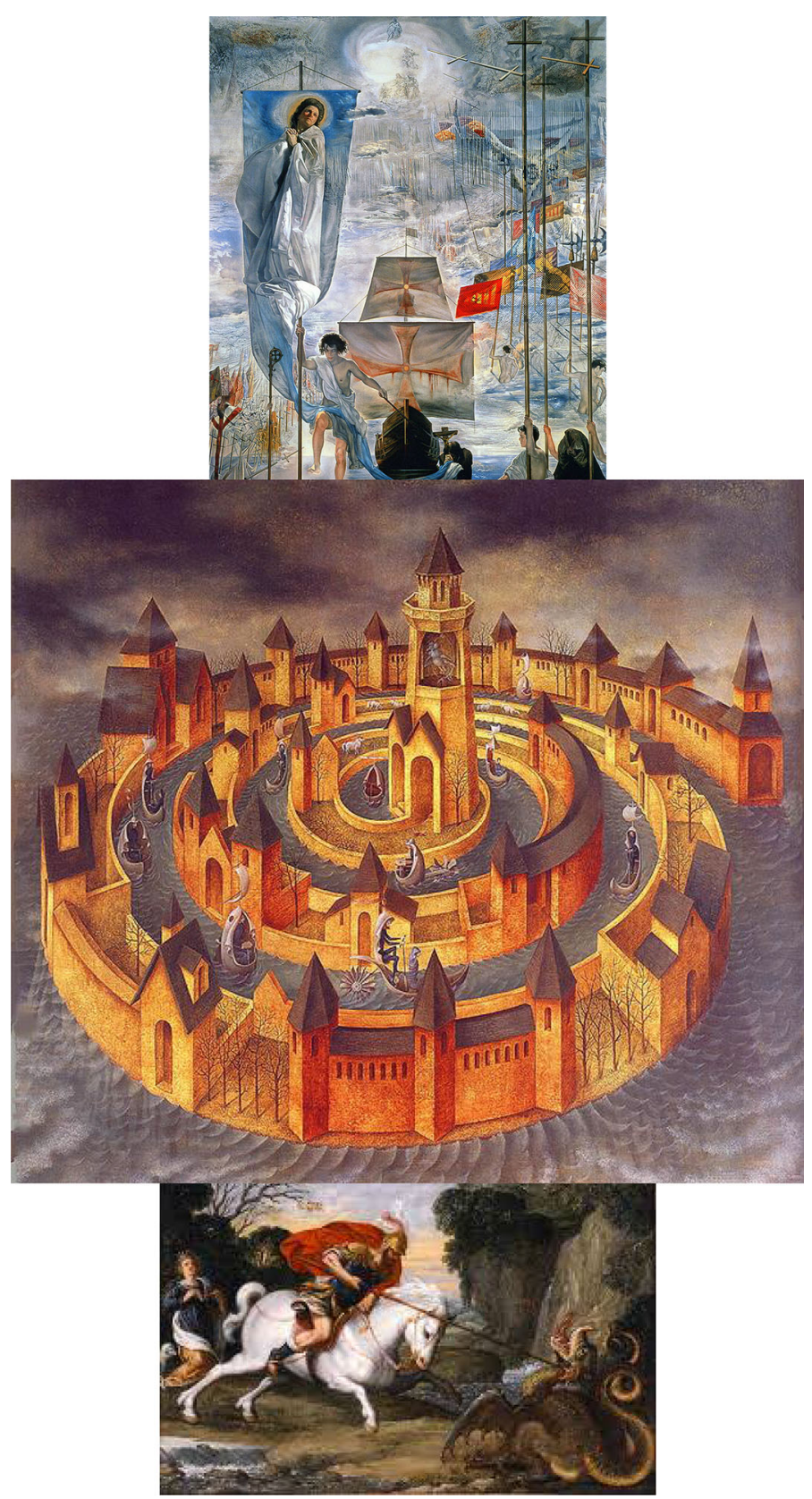

Fig. 2 Javier's Minneapolis triptych

\section{Static condensation and hybridization: devising HDG methods}

We present the HDG methods, not as they were originally introduced in 2009 [20], but by closely following the more didactic narrative which can be found in the 2016 review [6], 

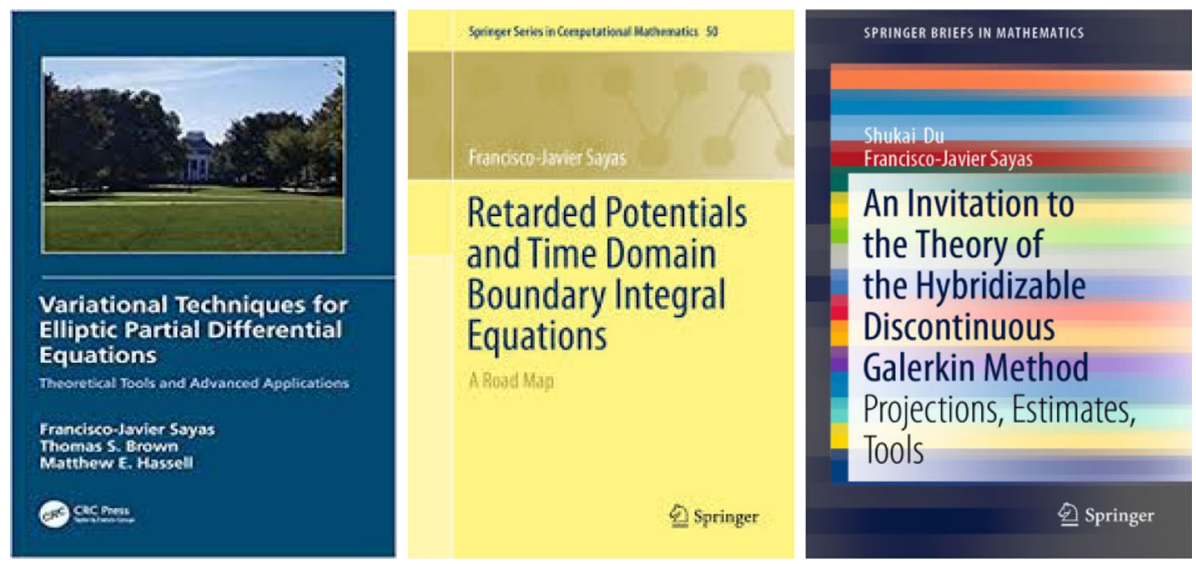

Fig. 3 Javier's Delaware triptych consists of his books on Variational Techniques (left), Boundary Integral methods (middle), and HDG methods (right)

as it merges the development of these methods with the evolution of the ideas of static condensation [47] and hybridization [42] introduced in 1965.

These two ideas were proposed in the framework of numerical methods for linear elasticity problems and were originally thought of as techniques for improving the matrices associated to the weak formulations of the methods, that is, for making them smaller and easier to numerically invert. Next, we show that we can also view those ideas as a single property of the exact solution which can then be used as a template to be applied to any of its approximations, like the continuous Galerkin and the mixed methods. Such template will be used for devising the HDG methods.

\subsection{The model problem and its exact solution}

We proceed in the framework of steady-state diffusion problems. So, we consider the following second-order elliptic model problem:

$$
\begin{aligned}
\mathrm{c} \boldsymbol{q}+\nabla u & =0 & & \text { in } \Omega, \\
\nabla \cdot \boldsymbol{q} & =f & & \text { in } \Omega, \\
\widehat{u} & =u_{D} & & \text { on } \partial \Omega .
\end{aligned}
$$

Here $\mathrm{c}$ is a matrix-valued function which is symmetric and uniformly positive definite on $\Omega$, $f$ is an $L^{2}(\Omega)$ function, and $\widehat{r}$ represents the trace operator.

For any element $K$ of a mesh $\mathcal{T}_{h}$ of the domain $\Omega$, we know that the exact solution satisfies the local problem

$$
\begin{aligned}
\mathrm{c} \boldsymbol{q}+\nabla u & =0 & & \text { in } K, \\
\nabla \cdot \boldsymbol{q} & =f & & \text { in } K .
\end{aligned}
$$

We also know that for any interior face $F=\partial K^{+} \cap \partial K^{-}, K^{+}, K^{-} \in \mathcal{T}_{h}$, it also satisfies the transmission conditions

$$
\begin{array}{rlr}
\widehat{u}^{+} \boldsymbol{n}^{+}+\widehat{u} & \boldsymbol{n}^{-}=: \llbracket \widehat{u} \rrbracket=0 & \text { on } F, \\
\widehat{\boldsymbol{q}}^{+} \cdot \boldsymbol{n}^{+}+\widehat{\boldsymbol{q}}^{-} \cdot \boldsymbol{n}^{-}=: \llbracket \widehat{\boldsymbol{q}} \rrbracket=0 & \text { on } F .
\end{array}
$$


Here, $\widehat{\eta}^{ \pm}$represents the trace, of the generic function $\eta$, on the face $F$ from the interior of the element $K^{ \pm}$, and $\boldsymbol{n}^{ \pm}$represents the unit normal to $F$ pointing outward from $K^{ \pm}$. Note that the first transmission condition is a reflection of the presence of the term $\nabla u$ in the first equation. The second transmission condition is a reflection of the presence of the term $\nabla \cdot \boldsymbol{q}$ in the second equation. Finally, we trivially know that the Dirichlet boundary condition

$$
\widehat{u}=u_{D} \quad \text { on } \partial \Omega,
$$

is also satisfied.

Conversely, suppose that we are given a sufficiently smooth function $\widehat{u}$ defined on $\mathcal{F}_{h}$, the set of all faces $F$ of the mesh $\mathcal{T}_{h}$. If for each element $K$ of the mesh $\mathcal{T}_{h}$, we define $(\boldsymbol{q}, u)$ in terms of $\widehat{u}$ and $f$ as the solution of the local problem

$$
\begin{aligned}
\mathrm{c} \boldsymbol{q}+\nabla u & =0 & & \text { in } K, \\
\nabla \cdot \boldsymbol{q} & =f & & \text { in } K, \\
u & =\widehat{u} & & \text { on } \partial K,
\end{aligned}
$$

we can then ask ourselves for what funcion $\widehat{u}$ we can say that $(\boldsymbol{q}, u)$ is the actual exact solution. The answer is that the function $\widehat{u}$ must be the solution of the global problem

$$
\begin{aligned}
\llbracket \widehat{\boldsymbol{q}} \rrbracket & =0 & & \text { if } F \text { is an interior face, } \\
\widehat{u} & =u_{D} & & \text { if } F \text { lies on } \partial \Omega,
\end{aligned}
$$

where $\widehat{\boldsymbol{q}}$ is the trace of $\boldsymbol{q}=\boldsymbol{q}(\widehat{u}, f)$ on $\partial K$. This is the property of the exact solution we alluded to above. Expressing $(\boldsymbol{q}, u)$ in terms of the unknown $\widehat{u}$ (and $f$ ) corresponds to the static condensation and the introduction of the unknown $\widehat{u}$ corresponds to the hybridization.

We can further refine this property by separating the influence of $\widehat{u}$ from that of $f$. Indeed, by the principle of superposition, we have that $(\boldsymbol{q}, u)=\left(\mathbf{Q}_{\widehat{u}}, \bigcup_{\widehat{u}}\right)+\left(\mathbf{Q}_{f}, \mathbf{U}_{f}\right)$, where

$$
\begin{aligned}
& \mathrm{c} \mathbf{Q}_{\widehat{u}}+\nabla \mathrm{U}_{\widehat{u}}=0 \quad \text { in } K, \quad \text { c } \mathbf{Q}_{f}+\nabla \mathbf{U}_{f}=0 \quad \text { in } K, \\
& \nabla \cdot \mathbf{Q}_{\widehat{u}}=0 \quad \text { in } K, \quad \nabla \cdot \mathbf{Q}_{f} \quad=f \quad \text { in } K, \\
& \mathrm{U}_{\widehat{u}}=\widehat{u} \quad \text { on } \partial K, \quad \mathrm{U}_{f} \quad=0 \quad \text { on } \partial K,
\end{aligned}
$$

and $\widehat{u}$ is the solution of the equations

$$
\begin{aligned}
-\llbracket \widehat{\mathbf{Q}}_{\widehat{u}} \rrbracket & =\llbracket \widehat{\mathbf{Q}}_{f} \rrbracket & & \text { on } F \in \mathcal{F}_{h}^{i}, \\
\widehat{u} & =u_{D} & & \text { on } \partial \Omega .
\end{aligned}
$$

Example Let us illustrate this characterization of the exact solution in the one-dimensional case $K=\left(x_{i-1}, x_{i}\right)$ for $i=1, \ldots, N$, with $\mathrm{c}=1$. We have that $(\boldsymbol{q}, u)=\left(\mathbf{Q}_{\widehat{u}}, \mathrm{U}_{\widehat{u}}\right)+$ $\left(\mathbf{Q}_{f}, \mathbf{U}_{f}\right)$, where

$$
\begin{aligned}
& \mathbf{Q}_{\widehat{u}}+\frac{d}{d x} \mathbf{U}_{\widehat{u}}=0 \quad \text { in }\left(x_{i-1}, x_{i}\right), \quad \mathbf{Q}_{f}+\frac{d}{d x} \mathbf{U}_{f}=0 \quad \text { in }\left(x_{i-1}, x_{i}\right), \\
& \frac{d}{d x} \mathbf{Q}_{\widehat{u}}=0 \quad \text { in }\left(x_{i-1}, x_{i}\right), \quad \frac{d}{d x} \mathbf{Q}_{f} \quad=f \quad \text { in }\left(x_{i-1}, x_{i}\right), \\
& \mathrm{U}_{\widehat{u}}=\widehat{u} \quad \text { on }\left\{x_{i-1}, x_{i}\right\}, \quad \mathrm{U}_{f} \quad=0 \quad \text { on }\left\{x_{i-1}, x_{i}\right\},
\end{aligned}
$$

and where the function $\widehat{u}$ is the solution of the global problem

$$
\begin{aligned}
\widehat{\mathbf{Q}}_{\widehat{u}}\left(x_{i}^{+}\right)-\widehat{\mathbf{Q}}_{\widehat{u}}\left(x_{i}^{-}\right) & =-\widehat{\mathbf{Q}}_{f}\left(x_{i}^{+}\right)+\widehat{\mathbf{Q}}_{f}\left(x_{i}^{-}\right) & & \text {for } i=1, \ldots, N-1, \\
\widehat{u}\left(x_{i}\right) & =u_{D}\left(x_{i}\right) & & \text { for } i=0, N .
\end{aligned}
$$


A simple computation gives us the solution of the local problem on $\left(x_{i-1}, x_{i}\right)$,

$$
\begin{array}{ll}
\mathbf{Q}_{\widehat{u}}(x)=-\frac{1}{h}\left(\widehat{u}_{i}-\widehat{u}_{i-1}\right), & \mathbf{Q}_{f}(x)=-\int_{x_{i-1}}^{x_{i}} G_{x}^{i}(x, s) f(s) d s, \\
\mathrm{U}_{\widehat{u}}(x)=\varphi_{i}(x) \widehat{u}_{i}+\varphi_{i-1}(x) \widehat{u}_{i-1}, & \mathrm{U}_{f}(x)=\int_{x_{i-1}}^{x_{i}} G^{i}(x, s) f(s) d s,
\end{array}
$$

where $G^{i}(\cdot, \cdot)$ is the Green's function of the local problem (see $\$ 2.1 .2$ in [6]), $G_{x}^{i}(\cdot, \cdot)$ its partial derivative with respect to the first variable, and $\varphi_{i}$ is the piecewise-linear function that is equal to one at $x_{i}$ and zero at $x_{j}$ for $j \neq i$. The function $\widehat{u}$ is then the solution of

$$
\begin{aligned}
-\frac{1}{h}\left(\widehat{u}_{i-1}-2 \widehat{u}_{i}+\widehat{u}_{i+1}\right) & =\int_{x_{i-1}}^{x_{i+1}} \varphi_{i}(s) f(s) d s & & \text { for } i=1, \ldots, N-1, \\
\widehat{u}\left(x_{i}\right) & =u_{D}\left(x_{i}\right) & & \text { for } i=0, N .
\end{aligned}
$$

Note that $\widehat{u}_{i}$ is nothing but the value of the exact solution at the partition point $x_{i}$. So the characterization of the exact solution shows that, if we can solve all the local problems exactly, the remaining values of the exact solution, $\widehat{u}$, can be obtained by solving a matrix equation.

\subsection{The continuous Galerkin method}

Now, let us apply the above template to the well-known continuous Galerkin method. This method provides an approximation to $u$, solution of our model problem rewritten as

$$
\begin{aligned}
-\nabla \cdot(\mathrm{a} \nabla u) & =f & & \text { in } \Omega, \\
\widehat{u} & =u_{D} & & \text { on } \partial \Omega,
\end{aligned}
$$

where $\mathrm{a}:=\mathrm{c}^{-1}$. Now, let us set

$$
\begin{aligned}
W_{h} & =\left\{w \in \mathcal{C}^{0}(\Omega):\left.w\right|_{K} \in W(K) \forall K \in \mathcal{T}_{h}\right\}, \\
W_{h}(g) & =\left\{w \in W_{h}:\left.w\right|_{\partial \Omega}=I_{h}(g)\right\},
\end{aligned}
$$

where, for each element $K \in \mathcal{T}_{h}, W(K)$ is a finite dimensional space (typically of polynomials), and $I_{h}$ is an interpolator or projection operator into the space of approximate traces $\left\{\left.w\right|_{\Gamma}: w \in W_{h}\right\}$. Then, we take the approximation $u_{h}$ as the element of $W_{h}\left(u_{D}\right)$ determined as the solution of the weak formulation

$$
\left(\mathrm{a} \nabla u_{h}, \nabla w\right)_{\Omega}=(f, w)_{\Omega} \quad \forall w \in W_{h}(0) .
$$

To apply the template, for each element $K \in \mathcal{T}_{h}$, we split the local space $W(K)$ as follows:

$$
\begin{aligned}
W(K) & =W_{0}(K) \oplus W_{\partial}(K), \\
W_{0}(K) & :=\left\{w \in W(K):\left.w\right|_{\partial K}=0\right\}, \\
W_{\partial}(K) & :=\left\{w \in W(K):\left.w\right|_{\partial K}=\left.0 \Longrightarrow w\right|_{K}=0\right\} .
\end{aligned}
$$

This induces the following split in the global spaces:

$$
\begin{aligned}
W_{h} & =W_{0, h} \oplus W_{\partial, h} \\
W_{0, h} & :=\left\{w \in W_{h}:\left.w\right|_{K} \in W_{0}(K) \forall K \in \mathcal{T}_{h}\right\}, \\
W_{\partial, h} & :=\left\{w \in W_{h}:\left.w\right|_{K} \in W_{\partial}(K) \forall K \in \mathcal{T}_{h}\right\},
\end{aligned}
$$


and allows us to rewrite the weak formulation defining $u_{h}$ as follows. First, we obtain $U \in$ $W(K)$ in terms of $\widehat{u}_{h}$ and $f$ by solving

$$
\begin{aligned}
(\mathrm{a} \nabla \mathrm{U}, \nabla w)_{K} & =(f, w)_{K} & & \forall w \in W_{0}(K), \\
\mathrm{U} & =\widehat{u}_{h} & & \text { on } \partial K .
\end{aligned}
$$

Then we have that $\mathrm{U}=u_{h}$ if and only if we take the function $\widehat{u}_{h}$ in $M_{h}\left(u_{D}\right)$, where

$$
\begin{aligned}
M_{h}(g) & :=\left\{\mu \in M_{h}:\left.\mu\right|_{\partial \Omega}=I_{h}(g)\right\}, \\
M_{h} & :=\left\{\left.w\right|_{\mathcal{F}_{h}}: w \in W_{h}\right\},
\end{aligned}
$$

and define it to be the solution of

$$
\begin{aligned}
(\mathrm{a} \nabla \mathrm{U}, \nabla w)_{\Omega} & =(f, w)_{\Omega} & & \forall w \in W_{\partial, h}(0), \\
\widehat{u}_{h} & =I_{h}\left(u_{D}\right) & & \text { on } \partial \Omega .
\end{aligned}
$$

The fact that the first of the equations determining $\widehat{u}_{h}$ can be interpreted as a transmission condition is proved in detail in [6]. There, we can also find the following further refinement of the above result. We have that $u_{h}=\mathrm{U}_{\widehat{u}_{h}}+\mathrm{U}_{f}$, where

$$
\begin{aligned}
\left(\mathrm{a} \nabla \mathbf{U}_{\mu}, \nabla w\right)_{K} & =0 & & \forall w \in W_{0}(K), \\
\mathbf{U}_{\mu} & =\mu & & \text { on } \partial K, \\
\left(\operatorname{a~} \nabla \mathbf{U}_{f}, \nabla w\right)_{K} & =(f, w)_{K} & & \forall w \in W_{0}(K), \\
\mathbf{U}_{f} & =0 & & \text { on } \partial K,
\end{aligned}
$$

and $\widehat{u}_{h}$ is the element of $M_{h}\left(u_{D}\right)$ that solves the global problem

$$
\left(\mathrm{a} \nabla \mathrm{U}_{\widehat{u}_{h}}, \nabla \mathrm{U}_{\mu}\right)_{\Omega}=\left(f, \mathrm{U}_{\mu}\right)_{\Omega} \quad \forall \mu \in M_{h}(0) .
$$

Relation with the static condensation technique by Guyan [47]. What we just did by using weak formulations, Guyan [47] did with matrices back in 1965. Let us establish a parallel between these two versions of the same procedure. The system of equations associated to the original weak formulation of the continuous Galerkin method is

$$
K\left[u_{h}\right]=[f] .
$$

After splitting the degrees of freedom, we can write that $u_{h}=U_{0}+U_{\partial}$, where $U_{\partial}$ is the element of $W_{\partial, h}$ such that $U_{\partial}=\widehat{u}_{h}$ on $\partial K$ for all $K \in \mathcal{T}_{h}$. Then the original equation becomes

$$
\left[\begin{array}{ll}
K_{00} & K_{0 \partial} \\
K_{\partial 0} & K_{\partial \partial}
\end{array}\right]\left[\begin{array}{l}
{\left[\mathrm{U}_{0}\right]} \\
{\left[\mathrm{U}_{\partial}\right]}
\end{array}\right]=\left[\begin{array}{l}
f_{0} \\
f_{\partial}
\end{array}\right] .
$$

The matrix form of the solution of the local problems is

$$
\left[\mathrm{U}_{0}\right]=-K_{00}^{-1} K_{0 \partial}\left[\mathrm{U}_{\partial}\right]+K_{00}^{-1}\left[f_{0}\right] .
$$

and the matrix form of the transmission condition is

$$
\left(-K_{\partial 0} K_{00}^{-1} K_{0 \partial}+K_{\partial \partial}\right)\left[\mathrm{U}_{\partial}\right]=-K_{\partial 0} K_{00}^{-1}\left[f_{0}\right]+\left[f_{\partial}\right] .
$$

Note that the process of static condensation just described in terms of matrices is nothing but a block-matrix Gauss elimination, and that the matrix associated to the transmission condition is nothing but the corresponding Schur complement matrix.

Note also that the unknown $\widehat{u}_{h}$ is completely captured by the part of the approximate solution we called $U_{\partial}$. This function already existed as part of the approximation and so 
the process of hybridization remained unnoticed. Indeed, no new, hybrid unknown had to be introduced. This is why only static condensation is attributed to Guyan [47]. The process of hybridization became evident in the framework of mixed methods, as we show next.

Example But before doing that, let us consider the application of the template to the one-dimensional case. For $W(K):=\mathcal{P}_{k}(K)$, the solutions of the local problems are

$$
\mathrm{U}_{\widehat{u}}(x)=\varphi_{i}(x) \widehat{u}_{i}+\varphi_{i-1}(x) \widehat{u}_{i-1} \quad \mathrm{U}_{f}(x)=\int_{x_{i-1}}^{x_{i}} G_{h}^{i}(x, s) f(s) d s,
$$

where $G_{h}^{i}(\cdot, \cdot)$ is the discrete Green's function (see \$2.2.4 in [6]), and the global problem for the values $\left\{\widehat{u}_{i}\right\}_{i=0}^{N}$ is

$$
\begin{aligned}
-\frac{1}{h}\left(\widehat{u}_{i-1}-2 \widehat{u}_{i}+\widehat{u}_{i+1}\right) & =\int_{x_{i-1}}^{x_{i+1}} \varphi_{i}(s) f(s) d s & & \text { for } i=1, \ldots, N-1, \\
\widehat{u}_{j} & =u_{D}\left(x_{j}\right) & & \text { for } j=0, N .
\end{aligned}
$$

The values $\left\{\widehat{u}_{i}\right\}_{i=0}^{N}$ are exact in this case as we can see if we compare this equation with the corresponding one for the exact solution.

\subsection{Mixed methods}

A mixed method defines an approximation to $(\boldsymbol{q}, u),\left(\boldsymbol{q}_{h}, u_{h}\right)$, as the only element of $\mathcal{V}_{h} \times W_{h}$ satisfying the equations

$$
\begin{aligned}
\left(\mathrm{c} \boldsymbol{q}_{h}, \boldsymbol{v}\right)_{\Omega}-\left(u_{h}, \nabla \cdot \boldsymbol{v}\right)_{\Omega} & =-\left\langle u_{D}, \boldsymbol{v} \cdot \boldsymbol{n}\right\rangle_{\partial \Omega} & & \forall \boldsymbol{v} \in \mathcal{V}_{h}, \\
\left(\nabla \cdot \boldsymbol{q}_{h}, w\right)_{\Omega} & =(f, w)_{\Omega} & & \forall w \in W_{h} .
\end{aligned}
$$

where

$$
\begin{array}{lll}
\mathcal{V}_{h}=\{\boldsymbol{v} \in \boldsymbol{H}(\operatorname{div}, \Omega): & \left.\boldsymbol{v}\right|_{K} \in \boldsymbol{V}(K) & \left.\forall K \in \mathcal{T}_{h}\right\}, \\
W_{h}=\left\{w \in L^{2}(\Omega):\right. & \left.w\right|_{K} \in W(K) & \left.\forall K \in \mathcal{T}_{h}\right\} .
\end{array}
$$

The scalar-valued functions in $W_{h}$ can be discontinuous across inter-element boundaries. However, the vector-valued functions in $\boldsymbol{V}_{h}$ must have continuous normal components across inter-element boundaries. We assume that, for every element $K \in \mathcal{T}_{h}$, the finite dimensional local spaces $\boldsymbol{V}(K)$ and $W(K)$ (typically of polynomials) have been chosen so that the method is well defined.

To apply the template under consideration to this method, we first have to hybridize the method by introducing a new unkown $\widehat{u}_{h}$ which we take in a suitably chosen space $M_{h}$. Only then we will be able to statically condense the method. To show how to do that, we follow [17].

We define $(\mathbf{Q}, \mathbf{U}) \in \boldsymbol{V}(K) \times W(K)$ in terms of $\widehat{u}_{h}$ and $f$ as the solution of the local problem

$$
\begin{aligned}
(\mathrm{c} \mathbf{Q}, \boldsymbol{v})_{K}-(\mathrm{U}, \nabla \cdot \boldsymbol{v})_{K} & =-\left\langle\widehat{u}_{h}, \boldsymbol{v} \cdot \boldsymbol{n}\right\rangle_{\partial K} & & \forall \boldsymbol{v} \in \boldsymbol{V}(K), \\
(\nabla \cdot \mathbf{Q}, w)_{K} & =(f, w)_{K} & & \forall w \in W(K) .
\end{aligned}
$$

Then, we take $\widehat{u}_{h}$ as the element of the space $M_{h}\left(u_{D}\right)$ satisfying

$$
\langle\llbracket \mathbf{Q} \rrbracket, \mu\rangle_{\mathcal{F}_{h}^{i}}=0 \quad \text { for all } \mu \in M_{h}(0) .
$$


Here, $M_{h}(g):=\left\{\mu \in M_{h}: \mu=g\right.$ on $\left.\partial \Omega\right\}$. Note that we can impose the Dirichlet boundary condition strongly since we do not take the restriction of $M_{h}$ to $\partial \Omega$ to lie in a finite dimensional space.

It is not difficult to show that this choice of $\widehat{u}_{h}$ is the only one which ensures that $(\mathbf{Q}, \mathrm{U})=$ $\left(\boldsymbol{q}_{h}, u_{h}\right)$, see [17] for details. Therein, it has also been proved that we can further refine the above characterization of the mixed method approximate solution as follows. We have that $\left(\boldsymbol{q}_{h}, u_{h}\right)=\left(\mathbf{Q}_{\widehat{u}_{h}}, \mathrm{U}_{\widehat{u}_{h}}\right)+\left(\mathbf{Q}_{f}, \mathrm{U}_{f}\right)$, where, $\forall K \in \mathcal{T}_{h}$,

$$
\begin{aligned}
\left(\mathrm{c} \mathbf{Q}_{\mu}, \boldsymbol{v}\right)_{K}-\left(\mathrm{U}_{\mu}, \nabla \cdot \boldsymbol{v}\right)_{K} & =-\langle\mu, \boldsymbol{v} \cdot \boldsymbol{n}\rangle_{\partial K} & & \forall \boldsymbol{v} \in \boldsymbol{V}(K), \\
\left(\nabla \cdot \mathbf{Q}_{\mu}, w\right)_{K} & =0 & & \forall w \in W(K), \\
\left(\mathrm{c} \mathbf{Q}_{f}, \boldsymbol{v}\right)_{K}-\left(\mathrm{U}_{f}, \nabla \cdot \boldsymbol{v}\right)_{K} & =0 & & \forall \boldsymbol{v} \in \boldsymbol{V}(K), \\
\left(\nabla \cdot \mathbf{Q}_{f}, w\right)_{K} & =(f, w)_{K} & & \forall w \in W(K),
\end{aligned}
$$

and the function $\widehat{u}_{h}$ is the element of $M_{h}\left(u_{D}\right)$ which solves the global problem

$$
\left(\mathrm{c} \mathbf{Q}_{\widehat{u}_{h}}, \mathbf{Q}_{\mu}\right)_{\mathcal{T}_{h}}=\left(f, \mathrm{U}_{\mu}\right) \mathcal{T}_{h} \quad \forall \mu \in M_{h}(0) \text {. }
$$

Here, $(\cdot, \cdot)_{\mathcal{T}_{h}}:=\sum_{K \in \mathcal{T}_{h}}(\cdot, \cdot)_{K}$.

\section{Relation with the hybridization of Fraejis de Veubeke [42]}

Let us now compare what we have done to what de Veubeke did back in 1965 [42] for a mixed method for linear elasticity.

The matrix equation of the mixed method is of the form

$$
\left[\begin{array}{ll}
\mathcal{A} & B \\
B^{t} & 0
\end{array}\right]\left[\begin{array}{l}
{\left[\boldsymbol{q}_{h}\right]} \\
{\left[u_{h}\right]}
\end{array}\right]=\left[\begin{array}{c}
-\left[u_{D}\right] \\
{[f]}
\end{array}\right] .
$$

Note that we define the vector $\left[u_{D}\right]$ by $[\boldsymbol{v}]^{t}\left[u_{D}\right]:=\left\langle\boldsymbol{v} \cdot \boldsymbol{n}, u_{D}\right\rangle_{\partial \Omega}$. Now, we hybridize the system by introducing the hybrid unknown $\widehat{u}_{h}$, by defining the matrix $C$ by $[v]^{t} C[\mu]:=$ $\langle\llbracket v \rrbracket, \mu\rangle_{\mathcal{F}_{h}^{i}}$, and finally by rewriting the original system as

$$
\left[\begin{array}{ccc}
A & B & C \\
B^{t} & 0 & 0 \\
C^{t} & 0 & 0
\end{array}\right]\left[\begin{array}{c}
{[\mathbf{Q}]} \\
{[\mathrm{U}]} \\
{\left[\widehat{u}_{h}\right]}
\end{array}\right]=\left[\begin{array}{c}
-\left[u_{D}\right] \\
{[f]} \\
0
\end{array}\right],
$$

where $\left[\widehat{u}_{h}\right]$ is the vector of the degrees of freedom of the hybrid unknown on the interior faces. This system of equations is significantly bigger than the original one, but is such that $(\mathbf{Q}, \mathbf{U})=\left(\boldsymbol{q}_{h}, u_{h}\right)$. Morever, by design, the local problems can be solved independently of each other. This means that the corresponding matrix, $\left[\begin{array}{cc}A & B \\ B^{t} & 0\end{array}\right]$, can be rearranged, by simple changes of rows and columns as a block-diagonal matrix and so, can be numerically inverted very easily. Thus, we can write that

$$
\left[\begin{array}{c}
{[\mathbf{Q}]} \\
{[\mathrm{U}]}
\end{array}\right]=\left[\begin{array}{cc}
A & B \\
B^{t} & 0
\end{array}\right]^{-1}\left[\begin{array}{c}
-C\left[\widehat{u}_{h}\right]-\left[u_{D}\right] \\
{[f]}
\end{array}\right],
$$

and eliminate the above unkowns from the equations, to get what is the matrix form of the transmission condition, namely,

$$
H\left[\widehat{u}_{h}\right]=H_{\partial}\left[u_{D}\right]+J[f],
$$


where $H:=C^{t}\left(A^{-1}-A^{-1} B\left(B^{t} A^{-1} B\right)^{-1} B^{t} A^{-1}\right) C$. Note, in contrast, the symplicity of the corresponding weak formulation!

Once again, we can see that the process of static condensation just described in terms of matrices is nothing but a block-matrix Gauss elimination, and that the matrix associated to the transmission condition is nothing but the corresponding Schur complement matrix.

Note also that the unknown $\widehat{u}_{h}$ was not part of the approximate solution of the mixed method. Its introduction marks the effective application of what is called hybridization. At first, it increases significantly the size of the matrix equation, but then it allows for an efficient static condensation as it results in a smaller (and symmetric, positive definite!) matrix for the hybrid unknown.

We are now ready to apply the template for the devising of the HDG methods. But before, let us quickly see how this process looks in the simple one dimensional case we have been considering.

Example Let us now show the mixed method in the one-dimensional case. For the wellknown Raviart-Thomas (RT) [56] space of index $k \geq 1$, namely, for $\boldsymbol{V}(K) \times W(K):=$ $\mathcal{P}_{k+1}(K) \times \mathcal{P}_{k}(K)$, the solutions of the local problems are

$$
\begin{array}{ll}
\mathbf{Q}_{\widehat{u}}(x)=-\frac{\widehat{u}_{i}-\widehat{u}_{i-1}}{h}, & \mathbf{Q}_{f}(x)=\int_{x_{i-1}}^{x_{i}} H_{h}^{i}(x, s) f(s) d s, \\
\mathbf{U}_{\widehat{u}}(x)=\varphi_{i}(x) \widehat{u}_{i}+\varphi_{i-1}(x) \widehat{u}_{i-1}, & \mathbf{U}_{f}(x)=\int_{x_{i-1}}^{x_{i}} G_{h}^{i}(x, s) f(s) d s,
\end{array}
$$

where $G_{h}^{i}(\cdot, \cdot)$ is the discrete local Green's function given by the method and $-H_{h}^{i}(\cdot, \cdot)$ its corresponding discrete $x$-derivative (see $\$ 2.3 .3$ in [6]), and the global problem for the values $\left\{\widehat{u}_{i}\right\}_{i=0}^{N}$ is

$$
\begin{aligned}
-\frac{1}{h}\left(\widehat{u}_{i-1}-2 \widehat{u}_{i}+\widehat{u}_{i+1}\right) & =\int_{x_{i-1}}^{x_{i+1}} \varphi_{i}(s) f(s) d s & & \text { for } i=1, \ldots, N-1, \\
\widehat{u}_{i} & =u_{D}\left(x_{j}\right) & & \text { for } i=0, N .
\end{aligned}
$$

Again the values $\left\{\widehat{u}_{i}\right\}_{i=0}^{N}$ are exact.

\subsection{HDG methods}

We now use the template to devise the HDG methods. The main advantage of this approach is that the static condensation and hybridization of the resulting methods are guaranteed by construction, see [20].

As we see next, the distinctive features of the HDG methods are that (i) the local problems are expressed in terms of discontinuous Galerkin methods, and that (ii) the transmission condition characterizing the hybrid unkown $\widehat{u}_{h}$ are nothing but the requirement (standard for discontinuous Galerkin methods) that the numerical flux be conservative.

So, on the element $K \in \mathcal{T}_{h}$, we define the approximation given by an HDG method, $\left(\boldsymbol{q}_{h}, u_{h}\right)$, in terms of $\left(\widehat{u}_{h}, f\right)$ as the element of $\boldsymbol{V}(K) \times W(K)$ which solves the discontinuous Galerkin (DG) scheme

$$
\begin{array}{clrl}
\left(\mathrm{c} \boldsymbol{q}_{h}, \boldsymbol{v}\right)_{K}- & \left(u_{h}, \nabla \cdot \boldsymbol{v}\right)_{K}+\left\langle\widehat{u}_{h}, \boldsymbol{v} \cdot \boldsymbol{n}\right\rangle_{\partial K}=0 & & \forall \boldsymbol{v} \in \boldsymbol{V}(K), \\
-\left(\boldsymbol{q}_{h}, \nabla w\right)_{K}+\left\langle\widehat{\boldsymbol{q}}_{h} \cdot \boldsymbol{n}, w\right\rangle_{\partial K}=(f, w)_{K} & & \forall w \in W(K), \\
\widehat{\boldsymbol{q}}_{h} \cdot \boldsymbol{n}=\boldsymbol{q}_{h} \cdot \boldsymbol{n}+\tau\left(u_{h}-\widehat{u}_{h}\right) & \text { on } \partial K .
\end{array}
$$


The hybrid unknown $\widehat{u}_{h}$ is defined as follows. For each face $F \in \mathcal{F}_{h}^{i}$, we take $\left.\widehat{u}_{h}\right|_{F}$ in a suitably defined finite dimensional space $M(F)$. Then, we determine $\widehat{u}_{h}$ as the solution of

$$
\begin{aligned}
& \left\langle\mu, \llbracket \widehat{\boldsymbol{q}}_{h} \rrbracket\right\rangle_{F}=0 \quad \forall \mu \in M(F) \quad \text { if } F \text { is an interior face, } \\
& \widehat{u}_{h}=u_{D} \quad \text { if } F \text { lies on } \partial \Omega .
\end{aligned}
$$

This completes the devising of the HDG methods by using this first template of the exact solution.

Note that, as wanted, the only globally-coupled degrees of freedom are those of the hybrid unknown, $\widehat{u}_{h}$. Note also that, for each interior face $F$, there are two values of the numerical flux $\widehat{\boldsymbol{q}}_{h}$. The transmission condition, that is, the first equation determining the hybrid unknown $\widehat{u}_{h}$, forces the normal components of those two values to weakly have the same value. In this way, a weak version of the local conservativity property, typical of DG methods, is imposed.

Suppose now that, on each face of an element, the stabilization function $\tau$ is the simple multiplication by the parameter $\tau$. Then, if the transmission condition implies that $\llbracket \widehat{\boldsymbol{q}}_{h} \rrbracket=0$, we have that

$$
\llbracket \boldsymbol{q}_{h} \rrbracket+\tau^{+}\left(u_{h}^{+}-\widehat{u}_{h}\right)+\tau^{-}\left(u_{h}^{-}-\widehat{u}_{h}\right)=0,
$$

which holds if

$$
\begin{aligned}
& \widehat{u}_{h}=\frac{\tau^{+} u_{h}^{+}+\tau^{-} u_{h}^{-}}{\tau^{+}+\tau^{-}}+\frac{1}{\tau^{+}+\tau^{-}} \llbracket \boldsymbol{q}_{h} \rrbracket, \\
& \widehat{\boldsymbol{q}}_{h}=\frac{\tau^{-} \boldsymbol{q}_{h}^{+}+\tau^{+} \boldsymbol{q}_{h}^{-}}{\tau^{+}+\tau^{-}}+\frac{\tau^{+} \tau^{-}}{\tau^{+}+\tau^{-}} \llbracket u_{h} \rrbracket
\end{aligned}
$$

provided $\tau^{+}+\tau^{-}>0$. These numerical traces are a particular case of the numerical traces of the DG methods considered in $[3,35]$. Those DG methods are hybridizable and hence amenable to an efficient static condensation. This is what gave the name HDG to these methods.

Note also that the hybrid function $\widehat{u}_{h}$ is both data for the local problems as well as the unknown for the global problem. The hybridization and static condensation approach used here to devise HDG methods for the steady-state diffusion problem can be extended to any partial differential equation by using what we could call a generalized hybridization and static condensation procedure. Indeed, the hybrid unknowns are nothing but any data which would render the local problems well posed. The global problem would then be the one that determines the hybrid unknowns. See the 2005 review [18] on hybridization techniques, and the 2009 paper [19] where HDG methods for the Stokes equations are devised by using four different choices of the hybrid unknowns.

As we did for the continuous Galerkin and the mixed methods, we can further refine the presentation of the HDG methods and rewrite them by separating the influence of $\widehat{u}_{h}$ from that of $f$. We can express the approximation $\left(\boldsymbol{q}_{h}, u_{h}\right)$ as the sum $\left(\mathbf{Q}_{\widehat{u}_{h}}, \mathbf{U}_{\widehat{u}_{h}}\right)+\left(\mathbf{Q}_{f}, \mathbf{U}_{f}\right)$, where, $\forall K \in \mathcal{T}_{h}$,

$$
\begin{aligned}
\left(\mathrm{c} \mathbf{Q}_{\mu}, \boldsymbol{v}\right)_{K}-\left(\mathrm{U}_{\mu}, \nabla \cdot \boldsymbol{v}\right)_{K} & =-\langle\mu, \boldsymbol{v} \cdot \boldsymbol{n}\rangle_{\partial K} & & \forall \boldsymbol{v} \in \boldsymbol{V}(K), \\
\left(\nabla \cdot \mathbf{Q}_{\mu}, w\right)_{K}+\left\langle\tau\left(\mathrm{U}_{\mu}-\mu\right), w\right\rangle_{\partial K} & =0 & & \forall w \in W(K), \\
\left(\mathrm{c} \mathbf{Q}_{f}, \boldsymbol{v}\right)_{K}-\left(\mathrm{U}_{f}, \nabla \cdot \boldsymbol{v}\right)_{K} & =0 & & \forall \boldsymbol{v} \in \boldsymbol{V}(K), \\
\left(\nabla \cdot \mathbf{Q}_{f}, w\right)_{K}+\left\langle\tau \mathrm{U}_{f}, w\right\rangle_{\partial K} & =(f, w)_{K} & & \forall w \in W(K),
\end{aligned}
$$

and the numerical trace $\widehat{u}_{h}$ is the element of the affine space

$$
M_{h}\left(u_{D}\right):=\left\{\mu \in L^{2}\left(\mathcal{F}_{h}\right):\left.\mu\right|_{F} \in M(F) \forall F \in \mathcal{F}_{h}^{i},\left.u_{h}\right|_{\partial \Omega}:=u_{D}\right\},
$$


satisfying the equations

$$
\left(c \mathbf{Q}_{\mu}, \mathbf{Q}_{\widehat{u}_{h}}\right)_{\mathcal{T}_{h}}+\left\langle\tau\left(\mathrm{U}_{\mu}-\mu\right),\left(\mathrm{U}_{\widehat{u}_{h}}-\widehat{u}_{h}\right)\right\rangle_{\partial \mathcal{T}_{h}}=\left(\mathrm{U}_{\mu}, f\right)_{\mathcal{T}_{h}} \quad \forall \mu \in M_{h}(0) .
$$

Here, $(\cdot, \cdot)_{\mathcal{T}_{h}}:=\sum_{K \in \mathcal{T}_{h}}(\cdot, \cdot)_{K}$ and $\langle\cdot, \cdot\rangle_{\partial \mathcal{T}_{h}}:=\sum_{K \in \mathcal{T}_{h}}\langle\cdot, \cdot\rangle_{\partial K}$. We can then see that the matrix equation for $\widehat{u}_{h}$ is symmetric. That it is also positive definite is easy to show when we assume that

(i) $\tau>0$ on $\partial K$,

(ii) $\nabla W(K) \subset V(K)$.

For a proof, see $[6,20]$. In sharp contrast with the mixed methods, note how simple is to choose the local spaces of the HDG methods.

The HDG methods inherit from the DG methods their ability of handling elements of arbitrary shape and arbitrary basis functions, as no strong inter-element continuity is required. The different HDG methods associated to this template, are generated by choosing the local spaces $\boldsymbol{V}(K), W(K), M(F)$ and the stabilization function $\tau$. Accordingly, two lines of research were immeditely generated which are still producing interesting results. One of them focuses on how to choose the local spaces whereas the other focuses on how to chose the stabilization functions.

\section{Javier and the evolution of the HDG methods}

\subsection{Superconvergent HDG methods}

Most of the work that Javier did on the HDG methods was on the line of research which emphasizes the choice of the local spaces. This approach takes advantage of the similarity of the formulations of the hybridized version of the mixed methods and the HDG methods. Indeed, note that, if in the latter formulation we set the stabilization function $\tau$ to zero, we do get a hybridized mixed method.

This is why a natural question to ask is what are the local spaces for which an HDG method has the same convergence properties of a mixed method? In particular, it is very well known that the RT method [56] for simplexes is such that a projection of the error $u-u_{h}$ converges with an additional power of $h$. Using this, one can devise an element-by-element post processing leading to a new approximation $u_{h}^{*}$ which converges with an additional power of $h$. That is why one says that this mixed method superconverges. The question now is if there are HDG methods which superconverge?

The first affirmative answer was given in 2008 [8] where the single face hybridizable (SFH) method was introduced. Defined on simplexes, the stabilization function was set to be zero on all faces of the simplex except on one, arbitrarily chosen. The second superconvergent HDG methods were found also in 2009 [24]. They use local discontinuous Galerkin (LDG) methods to define the local problems, are applied on meshes made of simplexes and use stabilization functions uniformily bounded above and below. This result was obtained by exploiting the above-mentioned similarity between the formulations of the HDG and the mixed methods.

In the 2010 paper [22], of which Javier was one of the authors, the first projection-based analysis of superconvergent HDG methods, defined on simplexes, was obtained. Such type of analysis was prompted by similar ones for the mixed methods. The mixed method projections ensure a powerful commuting diagram property which was thought to be necessary for their superconvergence property. The result in [22] showed that superconvergence can take place 
without such commuting diagram property. From this point on, all analyses of HDG methods would be projection-based.

Javier also participated in the extention of this projection-based technique for the analysis of superconvergent HDG methods for the Stokes flow of incompressible flow in 2011 [21] and again in 2014 [30] where the much more difficult case of exactly divergence-free approximate velocities was treated. The projection-based analysis was also extended for HDG methods for Timoshenko beams in 2012 [4].

All the above superconvergent HDG methods were obtained for simplicial elements. The question was now if there were superconvergent HDG methods defined in elements of arbitrary shape. The search for sufficient conditions for an HDG method to be superconvergent was carried out in 2012 [26,27], for steady-state diffusion problems. The extension of the projection-based analysis proposed in [22] was caried out to all these methods, and new superconvergent HDG methods for squares, cubes and prisms were constructed. The extension of this result to steady-state linear elasticity was done in 2013 in [33], and to the Stokes equations of incompressible flow also in 2013 in [32]; see the 2014 review [34].

At this point, no superconvergent HDG method for quadrilaterals or general, flat-faced hexahedra had been constructed. The work we just mentioned on the sufficient conditions for superconvergence had to be slighly tightened up for that to take place. So, in 2017, a theory of M-decompositions was introduced [14]. Javier did participate in its creation. Its application to the construction of superconvergent HDG methods defined on general polygonal elements was carried out in 2017 in [9] and for superconvergent HDG methods defined on general pyramids, prisms, and hexahedra also in 2017 in [10]. The extension to Stokes flow was done in 2017 in [13], and to elasticity in 2018 in [12]. The theory of M-decompositions can deliver new superconvergent mixed methods as it can construct superconvergent HDG methods. So, it can also be considered as a technique for constructing mixed methods for elements of arbitrary shape. As such, it can be used for the systematic construction of commuting exact sequences on elements of the above-mentioned shapes, see the 2017 paper [11]. For an introductory overview of the theory of M-decompositions, see [15].

\subsection{Coupling HDG and boundary element methods}

During his Zaragoza period, Javier had been interested in the coupling of LDG and BE methods $[2,45,46]$. During his Minneapolis period, he continued to develop this interest in three papers published in 2012, namely, on the coupling of the RT or HDG with BE methods [23], on general symmetric couplings of DG and BE methods [29], and on coupling at a distance HDG and BE methods [31]. During his Delaware period, he worked on nonsymmetric couplings of HDG and BE methods in the 2017 paper [44].

\subsection{Wave propagation}

The first HDG methods for the acoustic and elastic wave equations were implicit and were proposed in 2011 [51]. In 2014, the semidiscrete version of the methods for the acoustic wave equation was proven to be uniformly-in-time superconvergent in [28]. In 2016, explicit HDG methods for wave propagation were considered in [61]. Also on that year, an overview of the state of art on HDG method for hyperbolic problems appeared [25] which describes the state of the art up to that time.

It is, more or less, around this time that Javier took an interest in HDG methods for wave propagation. Javier developed MATLAB software for the HDG method in 3D, see the 
2015 paper [43], which was used, for example, in his 2018 paper on viscoelastic waves [1]. During that period, he also became interested in the application of HDG methods to wave propagation phenomena. Javier worked on the Stormer-Numerov HDG methods for the wave equation which was published in 2018 [16]. This was the last paper on which we collaborated. The results of the paper prompted the introduction of the Symplectic-Hamiltonian HDG methods: First for the acoustic wave equation, in 2017 [57], and then for the equations of elastodynamics, in 2021 [58]. Javier developed HDG methods for the time-harmonic linear elasticity in 2017 [48].

\subsection{Unified analyses, new stabilizations}

Javier also did work on the other main line of research on HDG methods, namely, the search for stabilization functions. In [49], see also [50], what is called the Lehrenfeld-Schöberl projection was introduced. It allows to define new HDG methods converging optimally regardless of the shape of the elements. The LDG-H methods also enjoy this property, but the spaces $M_{h}$ allowed with the use of the above-mentioned projection are smaller. In 2015 [54] we can find an analysis of the corresponding methods for steady-state diffusion; see also [55] the case of Stokes flow.

Another contribution in this line of research is the introduction of the hybrid high-oder (HHO) methods in 2014 [36,37]. For these methods, optimal convergence and superconvergence properties are achieved for elements of general shape. In 2016 [7] it was shown that the HHO method can be rewritten as an HDG method with a special stabilization. A distinctive role of this stabilization is to provide a higher-order of accuracy which the standard stabilizations do not provide.

Javier became interested in unifying the analyses of the HDG methods using the old stabilizations and the HDG using the Lehrenfled-Schöberl stabilization, which he called the HDG+ methods. He worked on a unified analysis of HDG methods for the steady-state Maxwell's equations in a paper published in 2020 [40]; notably, it included the HDG+ method introduced in 2017 in [5]. Also published in 2020, the paper [39] presents a single, simple and concise analysis of several variants of the HDG+ method using a projection-based approach. An interesting novelty here is that the theory of M-decompositions is used, but only as a intermediate step. Javier thus brought together the two lines of research on HDG methods. Finally, published in 2021 is a paper [41] in which a simple way of constructing HDG+ projections on polyhedral elements is introduced. This was Javier's last paper on HDG methods.

\section{Epilogue: the little poem of things that statically condense}

Javier's legacy not only lies in the many papers he wrote and the many Ph.D. Students he graduated. He touched many of us by his willingness to be actively engaged with the mathematical community. The yearly DelMar (Delaware-Maryland) Numerics Seminar started in 2012 and was originally organized by Ricardo H. Nochetto (University of Maryland), Petr Plechac and Francisco-Javier Sayas (University of Delaware), and by Tobias von Petersdorff (University of Maryland). It is thus fitting that, in memory of Javier, this seminar is now called the Sayas Numerical Day.

Moreover, the Sayas Numerics Seminar is a weekly online seminar which emphasizes, as Javier would have wanted, the participation of early career researchers. It has run during 
Fig. 4 The little poem of things that statically condense. The poem is traditionaly, but wrongly, attributed to Moments of the Soul, Monastery of Bgheno-Noravank in Syunik, 989. Javier's picture is taken from [52]

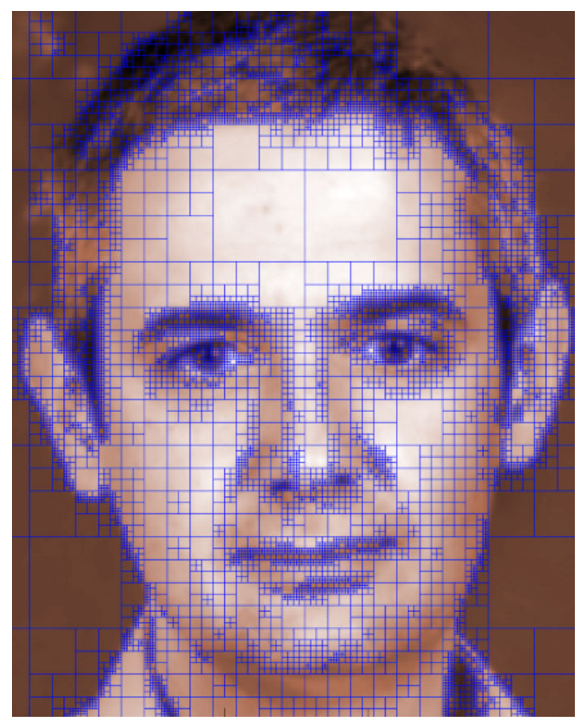

What does statically condense?

When implementing HDG methods, the degrees of freedom,

When drinking in a seminar BBQ, dew-like drops on a beer,

When saying farewell to a friend, tears on the heart.

the Fall semester of 2020 and the Spring semester of 2021, and I foresee it running many more semesters to come. Its organizers are Harbir Antil (George Mason University), Andrei Draganescu (University of Maryland), Ricardo H. Nochetto, Petr Plechac and Tobias von Petersdorff. The picture in Fig. 4 is the one that appears on the corresponding webpage. Its creation was instigated by Ricardo Nochetto [53] and carried out by Tobias von Petersdorff [62] by taking Javier's picture from the obituary on the University of Delaware website and then applying on it an adaptivity Matlab code he wrote.

To me, Javier was first a guest, then a collaborator and finally a friend. I want to leave in writing, verba volant, scripta manent, what I said to him on Saturday February 16th, 2019, in his meeting Numerical Analysis \& No Regrets:

Farewell Javier! It was good to play with you.

To end, let me summarize in Fig. 4 the story told in this short paper with a poem for Javier I read on that Saturday: The little poem of things that statically condense. We joked about the attribution. He then told me he liked the poem, accused me of being emotional, and gave me a big hug.

Acknowledgements I would like to thank Salim Meddahi for suggesting to the Scientific Committee of CEDYA my name for giving a talk in the tribute to Javier he organized, and to Peter Monk, member of the Scientific Committee of CEDYA, for supporting the nomination. I would also thank Mariano Mateos, Congress 
Chairman, for the invitation to participate in the XXVI CEDYA/ XVI CMA, June 14-18, 2021. CEDYA stands for Congreso de Ecuaciones Diferenciales y Aplicaciones, and CMA for Congreso de Matemática Aplicada. Concerning the paper itself, let me thank the referees for their constructive comments, and Gabriel Gatica for completing the information I had on the top panel of the first of Javier's triptychs. I would also like to thank Ricardo Nochetto and Tobias von Petersdorff for letting me use their figure of Javier [52] which I am displaying in Fig. 4. Thanks are due too to Scot Adams, Yanlai Chen, Shukai Du and Salim Meddahi for their sharp proofreading, and then again to Shukai Du for help in properly citing some of his work with Javier. Last but not least, I would like to thank Rosario Grau for her lucid editorial remarks.

Open Access This article is licensed under a Creative Commons Attribution 4.0 International License, which permits use, sharing, adaptation, distribution and reproduction in any medium or format, as long as you give appropriate credit to the original author(s) and the source, provide a link to the Creative Commons licence, and indicate if changes were made. The images or other third party material in this article are included in the article's Creative Commons licence, unless indicated otherwise in a credit line to the material. If material is not included in the article's Creative Commons licence and your intended use is not permitted by statutory regulation or exceeds the permitted use, you will need to obtain permission directly from the copyright holder. To view a copy of this licence, visit http://creativecommons.org/licenses/by/4.0/.

\section{References}

1. Brown, T., Du, S., Eruslu, H., Sayas, F.J.: Analysis of models for viscoelastic wave propagation. Appl. Math. Nonlinear Sci. 3, 55-96 (2018)

2. Bustinza, R., Gatica, G.N., Sayas, F.J.: A LDG-BEM coupling for a class of nonlinear exterior transmission problems. In: Numerical Mathematics and Advanced Applications, pp. 1129-1136. Springer, Berlin (2006)

3. Castillo, P., Cockburn, B., Perugia, I., Schötzau, D.: An a priori error analysis of the local discontinuous Galerkin method for elliptic problems. SIAM J. Numer. Anal. 38, 1676-1706 (2000)

4. Celiker, F., Cockburn, B., Shi, K.: A projection-based error analysis of HDG methods for Timoshenko beams. Math. Comp. 81, 131-151 (2012)

5. Chen, H., Qiu, W., Shi, K., Solano, M.: A superconvergent HDG method for the Maxwell equations. J. Sci. Comput. 70, 1010-1029 (2017)

6. Cockburn, B.: Static condensation, hybridization, and the devising of the HDG methods. In: Barrenechea, G.R., Brezzi, F., Cagniani, A., Georgoulis, E.H. (eds.) Building Bridges: Connections and Challenges in Modern Approaches to Numerical Partial Differential Equations, volume 114 of Lect. Notes Comput. Sci. Engrg., pp. 129-177. Springer, Berlin, 2016. LMS Durham Symposia funded by the London Mathematical Society. Durham, U.K., on July 8-16 (2014)

7. Cockburn, B., Di-Pietro, D.A., Ern, A.: Bridging the hybrid high-order and hybridizable discontinuous Galerkin methods. ESAIM Math. Model. Numer. Anal. 50, 635-650 (2016)

8. Cockburn, B., Dong, B., Guzmán, J.: A superconvergent LDG-hybridizable Galerkin method for secondorder elliptic problems. Math. Comp. 77, 1887-1916 (2008)

9. Cockburn, B., Fu, G.: Superconvergence by $M$-decompositions. Part II: construction of two-dimensional finite elements. ESAIM Math. Model. Numer. Anal 51(1), 165-186 (2017)

10. Cockburn, B., Fu, G.: Superconvergence by $M$-decompositions. Part III: construction of three-dimensional finite elements. ESAIM Math. Model. Numer. Anal 51(1), 365-398 (2017)

11. Cockburn, B., Fu, G.: A systematic construction of finite element commuting exact sequences. SIAM J. Numer. Anal. 55(4), 1650-1688 (2017)

12. Cockburn, B., Fu, G.: Devising superconvergent HDG methods with symmetric approximate stresses for linear elasticity by M-decompositions. IMA J. Numer. Anal. 38(2), 566-604 (2018)

13. Cockburn, B., Fu, G., Qiu, W.: A note on the devising of superconvergent HDG methods for Stokes flow by $M$-decompositions. IMA J. Numer. Anal. 37(2), 730-749 (2017)

14. Cockburn, B., Fu, G., Sayas, F.J.: Superconvergence by $M$-decompositions. Part I: general theory for HDG methods for diffusion. Math. Comput. 86(306), 1609-1641 (2017)

15. Cockburn, B., Fu, G., Shi, K.: An introduction to the theory of $M$-decompositions. In: Numerical methods for PDEs, vol. 15 of SEMA SIMAI Springer Ser., pp. 5-29. Springer, Cham (2018)

16. Cockburn, B., Fu, Z., Hungria, A., Ji, L., Sánchez, M.A., Sayas, F.J.: Stormer-Numerov HDG methods for acoustic waves. J. Sci. Comput. 75(2), 597-624 (2018)

17. Cockburn, B., Gopalakrishnan, J.: A characterization of hybridized mixed methods for second order elliptic problems. SIAM J. Numer. Anal. 42, 283-301 (2004) 
18. Cockburn, B., Gopalakrishnan, J.: New hybridization techniques. GAMM MitT. Ges. Angew. Math. Mech. 2, 154-183 (2005)

19. Cockburn, B., Gopalakrishnan, J.: The derivation of hybridizable discontinuous Galerkin methods for Stokes flow. SIAM J. Numer. Anal. 47, 1092-1125 (2009)

20. Cockburn, B., Gopalakrishnan, J., Lazarov, R.: Unified hybridization of discontinuous Galerkin, mixed and continuous Galerkin methods for second order elliptic problems. SIAM J. Numer. Anal. 47, 13191365 (2009)

21. Cockburn, B., Gopalakrishnan, J., Nguyen, N.C., Peraire, J., Sayas, F.J.: Analysis of an HDG method for Stokes flow. Math. Comput. 80, 723-760 (2011)

22. Cockburn, B., Gopalakrishnan, J., Sayas, F.J.: A projection-based error analysis of HDG methods. Math. Comput. 79, 1351-1367 (2010)

23. Cockburn, B., Guzmán, J., Sayas, F.J.: Coupling of Raviart-Thomas and hybridizable discontinuous Galerkin methods with BEM. SIAM J. Numer. Anal. 50, 2778-2801 (2012)

24. Cockburn, B., Guzmán, J., Wang, H.: Superconvergent discontinuous Galerkin methods for second-order elliptic problems. Math. Comput. 78, 1-24 (2009)

25. Cockburn, B., Nguyen, N.C., Peraire, J.: HDG methods for hyperbolic problems. In: Handbook of Numerical Methods for Hyperbolic Problems, vol. 17 of Handb. Numer. Anal., pp. 173-197. Elsevier/North-Holland, Amsterdam (2016)

26. Cockburn, B., Qiu, W., Shi, K.: Conditions for superconvergence of HDG methods for second-order eliptic problems. Math. Comput. 81, 1327-1353 (2012)

27. Cockburn, B., Qiu, W., Shi, K.: Conditions for superconvergence of HDG methods on curvilinear elements for second-order eliptic problems. SIAM J. Numer. Anal. 50, 1417-1432 (2012)

28. Cockburn, B., Quenneville-Bélair, V.: Uniform-in-time superconvergence of HDG methods for the acoustic wave equation. Math. Comput. 83, 65-85 (2014)

29. Cockburn, B., Sayas, F.J.: The devising of symmetric couplings of boundary element and discontinuous Galerkin methods. IMA J. Numer. Anal. 32, 765-794 (2012)

30. Cockburn, B., Sayas, F.J.: Divergence-conforming HDG methods for Stokes flow. Math. Comput. 83, $1571-1598(2014)$

31. Cockburn, B., Sayas, F.J., Solano, M.: Coupling at a distance HDG and BEM. SIAM J. Sci. Comput. 34, A28-A47 (2012)

32. Cockburn, B., Shi, K.: Conditions for superconvergence of HDG methods for Stokes flow. Math. Comput. 82, 651-671 (2013)

33. Cockburn, B., Shi, K.: Superconvergent HDG methods for linear elasticity with weakly symmetric stresses. IMA J. Numer. Anal. 33, 747-770 (2013)

34. Cockburn, B., Shi, K.: Devising HDG methods for Stokes flow: an overview. Comput. Fluids 98, 221-229 (2014)

35. Cockburn, B., Shu, C.-W.: The local discontinuous Galerkin method for time-dependent convectiondiffusion systems. SIAM J. Numer. Anal. 35, 2440-2463 (1998)

36. Di-Pietro, D.A., Ern, A.: A hybrid high-order locking-free method for linear elasticity on general meshes. Comput. Method Appl. Mech. Eng. 283, 1-21 (2015)

37. Di-Pietro, D.A., Ern, A., Lemaire, S.: An arbitrary-order and compact-stencil discretization of diffusion on general meshes based on local reconstruction operators. Comput. Method. Appl. Math. 14(4), 461-472 (2014)

38. Du, S., Sayas, F.J.: An invitation to the theory of the hybridizable discontinuous Galerkin method: projections, estimates, tools. SpringerBriefs in Mathematics. Springer, Berlin (2019)

39. Du, S., Sayas, F.J.: New analytical tools for HDG in elasticity, with applications to elastodynamics. Math. Comput. 89, 1745-1782 (2020)

40. Du, S., Sayas, F.J.: A unified analysis of HDG methods for the static Maxwell equations. SIAM J. Numer. Anal. 58, 1367-1391 (2020)

41. Du, S., Sayas, F.J.: A note on devising HDG+ projections on polyhedral elements. Math. Comput. 90, 65-79 (2021)

42. Fraejis de Veubeke, B.M.: Displacement and equilibrium models in the finite element method. In: Zienkiewicz, O.C., Holister, G. (eds.) Stress Analysis, pp. 145-197. Wiley, New York (1977)

43. Fu, Z., Gatica, L.F., Sayas, F.J.: Algorithm 949:MATLAB tools for HDG in three dimensions. ACM Trans. Math. Softw. 69, 620-635 (2015)

44. Fu, Z., Heuer, N., Sayas, F.J.: A non-symmetric coupling of boundary element methods with the hybridizable discontinuous Galerkin method. Comput. Math. Appl. 74, 2752-2768 (2017)

45. Gatica, G.N., Heuer, N., Sayas, F.J.: A direct coupling of local discontinuous Galerkin and boundary element methods. Math. Comput. 79, 1369-1394 (2010) 
46. Gatica, G.N., Sayas, F.J.: An a priori error analysis for the coupling of local discontinuous Galerkin and boundary element methods. Math. Comput. 75(256), 1675-1696 (2006). (electronic)

47. Guyan, R.J.: Reduction of stiffness and mass matrices. J. Am. Inst. Aron. Astrol. 3, 380 (1965)

48. Hungria, A., Prada, D., Sayas, F.J.: HDG methods for elastodynamics. Comput. Math. Appl. 74, 26712690 (2017)

49. Lehrenfeld, C.: Hybrid discontinuous Galerkin methods for solving incompressible flow problems. PhD thesis, Diplomigenieur Rheinisch-Westfalishen Technischen Hochchule Aachen (2010)

50. Lehrenfeld, C., Schöberl, J.: High order exactly divergence-free hybrid discontinuous Galerkin methods for unsteady incompressible flows. Comput. Methods Appl. Mech. Eng. 307, 339-361 (2016)

51. Nguyen, N.C., Peraire, J., Cockburn, B.: High-order implicit hybridizable discontinuous Galerkin methods for acoustics and elastodynamics. J. Comput. Phys. 230, 3695-3718 (2011)

52. Nochetto, R., von Petersdorff, T.: Sayas numerics seminar, online seminar on computational mathematics. 2020. https://sayasseminar.math.umd.edu/. Accessed July 2021

53. Nochetto, R.H.: Private communication. (2021)

54. Oikawa, I.: A hybridized discontinuous Galerkin method with reduced stabilization. J. Sci. Comput. 65, 327-340 (2015)

55. Oikawa, I.: Analysis of a reduced-order HDG method for the Stokes equations. J. Sci. Comput. 67(2), 475-492 (2016)

56. Raviart, P.A., Thomas, J.M.: A mixed finite element method for second order elliptic problems. In: Galligani, I., Magenes, E. (eds.) Mathematical Aspects of Finite Element Method. Lecture Notes in Math, pp. 292-315. Springer, New York (1977)

57. Sánchez, M.A., Ciuca, C., Nguyen, N.C., Peraire, J., Cockburn, B.: Symplectic Hamiltonian HDG methods for wave propagation phenomena. J. Comput. Phys. 350, 951-973 (2017)

58. Sánchez, M.A., Cockburn, B., Nguyen, N.-C., Peraire, J.: Symplectic Hamiltonian finite element methods for linear elastodynamics. Comput. Methods Appl. Mech. Eng 381, 113843 (2021)

59. Sayas, F.J.: The validity of Johnson-Nédélec's BEM-FEM coupling on polygonal interfaces. SIAM J. Numer. Anal. 47(5), 3451-3463 (2009)

60. Sayas, F.J.: The validity of Johnson-Nédélec's BEM-FEM coupling on polygonal interfaces. SIAM Rev. 55(1), 131-146 (2016)

61. Stanglmeier, M., Nguyen, N.-C., Peraire, J., Cockburn, B.: An explicit hybridizable discontinuous Galerkin method for the acoustic wave equation. Comput. Methods Appl. Mech. Eng. 300, 748-769 (2016)

62. von Petersdorff, T.: Private communication. (2021)

Publisher's Note Springer Nature remains neutral with regard to jurisdictional claims in published maps and institutional affiliations. 